\title{
Inline-Prozessüberwachung von Suspensionen mit hohem Feststoffanteil
}

\author{
S. Wöckel ${ }^{1}$, U. Steinmann ${ }^{1}$, R. Weser ${ }^{2}$, B. Wessely ${ }^{2}$ \\ ${ }^{1}$ Institut für Automation und Kommunikation (ifak) e.V. Magdeburg, Werner-Heisenberg-Str. 1, 39106 \\ Magdeburg, Deutschland \\ sebastian.woeckel@ifak.eu \\ ${ }^{2}$ Technische Universität Dresden, Institut für Verfahrenstechnik und Umwelttechnik, Arbeitsgruppe \\ Mechanische Verfahrenstechnik, 01062 Dresden, Deutschland
}

\section{Zusammenfassung}

Es wurde ein neuartiges Ultraschallmessverfahren entwickelt, mit dem die akustischen Streusignale aus hochkonzentrierten Suspensionen erfasst und zum Zweck der Partikelcharakterisierung ausgewertet werden können. Der Sensor ist als Einstecksonde konzipiert und erlaubt eine schnelle, einfache und minimalinvasive Prozessankopplung. Anstelle eines Einzelsignals im Zeitbereich wird ein statistischer Kennwert (Standardabweichung $\eta(t)$ ) aus einer Anzahl $N$ von Einzelmessungen der laufzeitabhängigen akustischen Rückstreusignale ermittelt. Dadurch kann neben der Schalldämpfung, die maßgeblich von der vorhandenen Partikelkonzentration beeinflusst wird, auch der Rückstreukoeffizient bestimmt werden, der sensitiv gegenüber der Partikelgröße ist. Diese Abhängigkeiten konnten durch experimentelle und theoretische Untersuchungen im Frequenzbereich von 5 bis $20 \mathrm{MHz}$, für Partikelgrößen von 1 bis $500 \mu \mathrm{m}$ und Partikelkonzentration bis zu $50 \mathrm{Ma}$.- \% belegt werden. Die Vermeidung eines Messspalts, wie im Fall der klassischen Transmissionsanordnung, prädestiniert das Messverfahrens für die Inline-Anwendung an sehr hochkonzentrierten Suspensionen.

Key words (5): Ultraschall, Partikelmessung, Reflexion, Suspension

\section{Motivation}

Eine Reihe von verfahrenstechnischen oder biotechnologischen Prozessen ist geprägt durch die Charakterisierung der Feststoffphase in hochkonzentrierten Suspensionen. So können die inline gewonnenen Daten zur Partikelgrößenverteilung und -konzentration dazu beitragen, die verschiedenen Prozesse optimal zu steuern, Produktqualitäten gezielt zu steigern oder Anlagenverfügbarkeiten zu erhöhen.

Eine der etablierten Methoden basiert auf der akustischen Durchschallung der Suspension und zeichnet sich durch eine schnelle, inlinefähige Arbeitsweise aus, die im Gegensatz zu optischen Methoden auch an hoch konzentrierten, opaken Proben ohne Verdünnung angewendet werden kann. Die Ultraschall(US)Technik ist auch unter rauen Prozessbedingungen einsetzbar und erlaubt den Einsatz in einem weiten Konzentrations- und Partikelgrößenbereich.

Als primäre Messinformation nutzt das Verfahren die frequenzabhängige US-Dämpfung der mit dem zu analysierenden Medium wechselwirkenden Ultraschallwelle. Daraus lassen sich
Informationen bezüglich der Dimension, Größenverteilung und Konzentration der Teilchen in Suspensionen und Emulsionen ableiten. Hierzu werden die US-Wandler häufig in einer sich gegenüberliegenden Sender-EmpfängerAnordnung positioniert. Aufgrund der erheblichen Schalldämpfung ist es jedoch bei hoch konzentrierten Stoffsystemen erforderlich, den Abstand zwischen beiden Wandlern hinreichend klein zu gestalten $(0,5 \mathrm{~mm}-2 \mathrm{~mm})$, um ein noch auswertbares Messsignal zu erhalten. Dies führt bei Inline-Anwendungen häufig zu Verstopfungen innerhalb der Messstrecke (vor allem beim Vorhandensein einzelner großer Feststoffpartikel oder pastöser Medien), was die Zuverlässigkeit und die Akzeptanz der Messeinrichtung bei den Anwendern einschränkt.

Folglich besteht eine nicht unerhebliche Forderung nach prozessfähiger und vergleichsweise kostengünstiger (Partikel-)Messtechnik, die auch unter Prozessbedingungen zuverlässig, stabil und rückwirkungsfrei arbeitet. Einen Erfolg versprechenden Ansatz sehen die Autoren in der Messung der von der Suspension gestreuten (statt transmittierten) Schallanteile in Abhängigkeit von der Frequenz. 


\section{Messprinzip und Versuchsaufbau}

Verschiedene minimalinvasive Sondenkonzepte wurden entwickelt und getestet, die entweder im Impuls-Echo-Modus mit einem einzelnen Ultraschallwandler arbeiten oder zwei unter einem Winkel angeordnete Ultraschallwandler im Sender-Empfänger-Modus nutzen (vgl. Abb. 1). Der grundlegende Aufbau einer Sonde beinhaltet den (die) Schallwandler, die Vorlaufstrecke (fest oder flüssig) sowie das akustische Fenster als Zwischenschicht zur Suspension. Die Vorlaufstrecke dient u.a. der Entkopplung der empfindlichen Wandleroberfläche(n) vom abrasiven Partikelsystem, der Gewährleistung der Umschaltzeiten inklusive Nachschwingungen des piezokeramischen Schallwandlers im Impuls-Echo-Verfahren und der Ausblendung des Nahfeldes.

Neben der Messsonde ist die Bereitstellung homogener, gleichverteilter sich bewegender Partikel entscheidend für die Funktionalität des Verfahrens. In diesem Zusammenhang wurden unterschiedliche Anordnungen hinsichtlich der nicht mit der Strömung korrelierten Gleichverteilung der Partikel in der Messzelle untersucht. Abb. 2 zeigt exemplarisch die Prinzipskizze einer geschlossenen Labormesszelle mit der ein Partikelgemisch hoher Konzentration blasenfrei mit verschiedenen Strömungsgeschwindigkeiten in Bewegung gehalten werden kann.

Als Anregungssignale und zu Gunsten einer höheren Ortsauflösung im Empfangssignal sind breitbandige Kurzzeitimpulse vorteilhaft, die

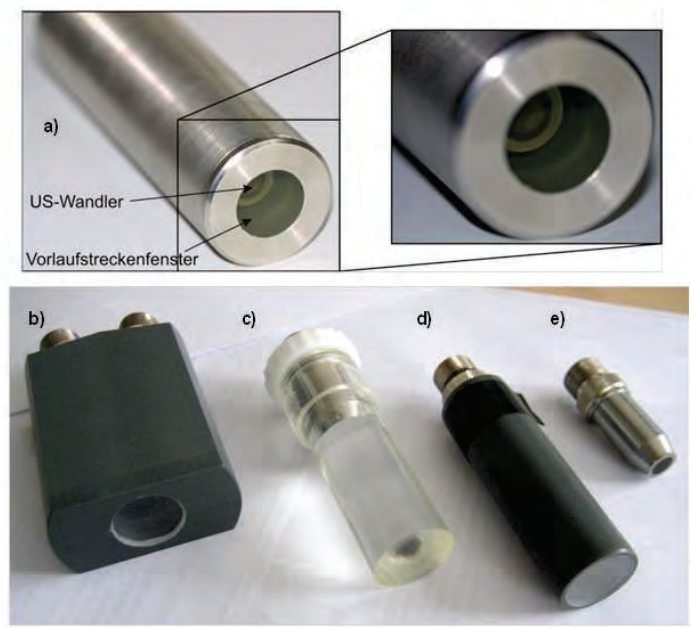

Abb. 1. Impuls-Echo-Sonde für Impulsbetrieb mit verkürzter Vorlaufstrecke (a), mit $50 \mathrm{~mm}$ fester Vorlaufstrecke (c) oder flüssiger Vorlaufstrecke (d). Die Sender-Empfänger-Sonde unter (b) hat einen Einschallwinkel von $12^{\circ}$ und $50 \mathrm{~mm}$-Vorlauf. Die Sonden $(a, b, d)$ nutzen ein akustisches Fenster $(d=1 \mathrm{~mm})$. Die Bauform der verwendeten ImmersionsUltraschallwandler (e) ist für alle Sonden gleich und austauschbar für den Frequenzbereich 2,25 MHz bis $25 \mathrm{MHz}$.

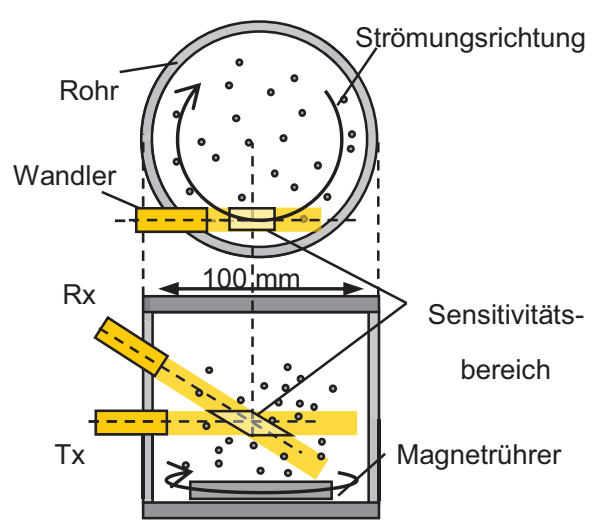

Abb. 2. Schematische Darstellung des Messvolumens für winkelabhängige Ultraschallstreuung (oben: Draufsicht, unten: Seitenansicht).

entweder konventionell durch kurze RechteckSpannungspulse oder über ein quasiperiodisch kodiertes Signal [1] synthetisch erzeugt werden.

\section{Signalanalyse}

Nach Anregung ist am Empfänger ein charakteristisches Rückstreusignal (Schalldruckamplitude $p(t)$ ) messbar, das neben den Reflexionen am Vorlaufstreckenfenster die interessierenden, aber sehr schwachen Rückstreusignale an den Partikeln enthält. In einer statistischen Auswertung wird die Standardabweichung zu jedem Abtastzeitpunkt aus den jeweils $N$ Amplitudenwerten ermittelt. Sie kann als Äquivalenzmaß für die Amplitude der Rückstreusignale $\eta(t)$ aufgefasst werden (Abb. 3). Diese Form der statistischen Signalauswertung erlaubt ein repräsentatives Abbild des gesamten Stoffsystems, da verschiedene Partikelkonstellationen und eine große Partikelanzahl durch die Zwangsbewegung (Rührer) in der Messzone erfasst werden.

Die vom Schallwandler detektierbare Rückstreusignalamplitude verringert sich aufgrund der mit der zurückgelegten Wegstrecke zunehmenden Dämpfung über die Laufzeit. Die Schallabschwächung wächst exponentiell mit der durchschallten Wegstrecke bzw. der Laufzeit an und wird $u$. a. von der Partikelgröße und -konzentration bestimmt [2, 3]. Wird für die Darstellung der Rückstreuamplitude eine logarithmische Skalierung gewählt, kann der Einfluss der Schalldämpfung linearisiert dargestellt und deren Verlauf über der Signallaufzeit mittels einer Geraden der Form $\eta(t)=-\Delta \eta \cdot t+\eta_{\max }$ beschrieben werden. Der Abfall der Geraden $(\Delta \eta)$ gibt die laufzeitabhängige Schallabschwächung in der Form Dezibel pro Zeiteinheit (z.B. dB/ $/ \mu s$ ) wieder. Der zweite Parameter der Geradengleichung $\left(\eta_{\max }\right)$ wird als derjenige 


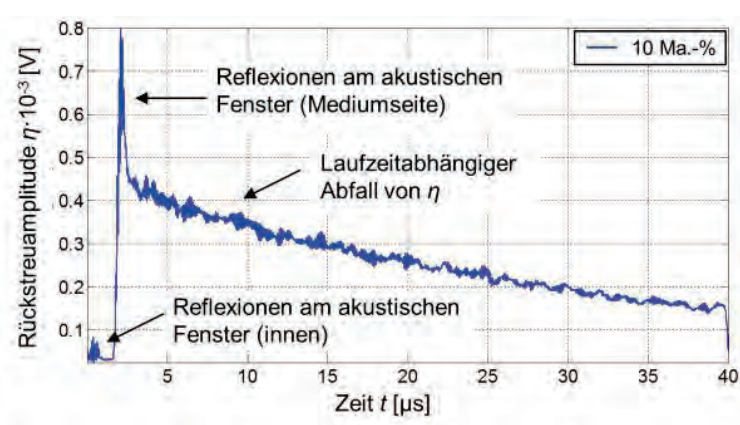

Abb. 3. Über $N=500$ Einzelmessungen ermittelte Rückstreuamplitude $\eta$ für eine wässrige Suspension mit $10 \mathrm{Ma} . \%$ CP3000 Partikeln ( $d_{50}=12 \mu \mathrm{m}, \mathrm{SiO}_{2}-$ Pigment der Firma Potters Ballotini).

Wert der Rückstreuamplitude definiert, der direkt nach dem Vorlaufstreckenfenster, also zu Beginn der Wechselwirkung zwischen der emittierten Schallwelle und dem Partikelsystem auftritt [4]. Eine vorherige Frequenzfilterung der Messdaten erlaubt zusätzlich die spektrale Bestimmung der beiden Fitparameter. Für den Fall einer Impulsanregung bestimmt die Bandbreite des verwendeten Wandlers den auswertbaren Frequenzbereich.

\section{Messergebnisse}

Die messtechnischen Untersuchungen konzentrierten sich zum einen auf Suspensionen mit veränderlicher Partikelkonzentration bei gleichbleibender Partikelgröße. Demgegenüber wurden weiterhin auch Messungen an Stoffsystemen konstanter Konzentration aber veränderlicher Partikelgröße durchgeführt.

Im Ergebnis der ersten durchgeführten Rückstreumessungen zeigt sich ein deutlicher Einfluss der Partikelkonzentration auf die Schallabschwächung $\Delta \eta$ (Abb. 4). Im untersuchten Konzentrationsbereich steigt die Schalldämpfung mit zunehmender Partikelanzahl im Messvolumen kontinuierlich an, wobei die Partikelgröße den Anstieg zusätzlich derart beeinflusst, dass sich dieser (im untersuchten Größenbereich) mit zunehmender Partikelgröße abschwächt.

Ein Vergleich der auf Basis der Rückstreumessung gewonnenen Schalldämpfungswerte mit jenen des in Transmission arbeitenden Ultraschall-Dämpfungsspektrometers DT1200 [5] als Referenzmessverfahren wurde zur Plausibilitätsprüfung der Messergebnisse herangezogen (Abb. 5). Es zeigt sich, dass die Messwertpaare entlang der Paritätslinie angeordnet sind. Die aus der relativen Änderung des Rückstreusignals bestimmten Schalldämpfungswerte sind

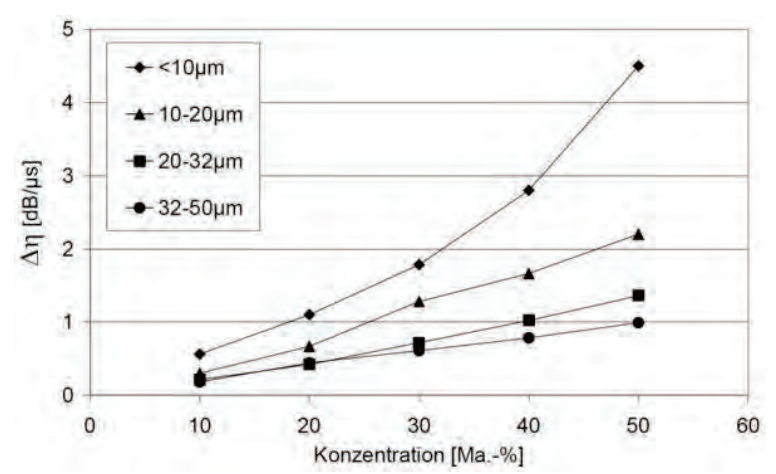

Abb. 4. Laufzeitabhängige Schallabschwächung $\Delta \eta$ als Funktion der Partikelkonzentration und bei verschiedenen Partikelgrößenfraktionen.

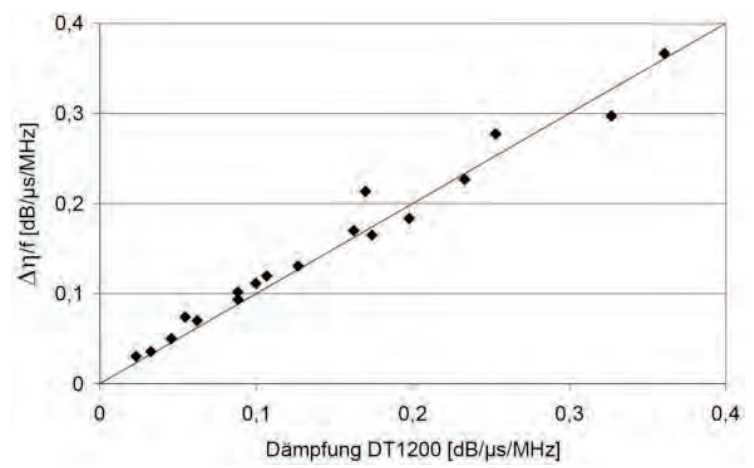

Abb. 5. Vergleich der gemessenen Schalldämpfungswerte in Rückstreuung ( $f=6 \mathrm{MHz}$ ) mit jenen des Referenz-Ultraschall-Dämpfungsspektrometers DT 1200.

folglich äquivalent den Referenzwerten aus der Transmissionsmessung.

Neben der Schalldämpfung liefert die Rückstreumessung mit dem Maximalwert der Rückstreuamplitude $\eta_{\max }$ einen weiteren Parameter. Abb. 6 zeigt den experimentell ermittelten Zusammenhang zwischen dieser Messgröße und der mittleren Partikelgröße der jeweiligen Partikelgrößenfraktion. Im untersuchten Partikelgrößenbereich steigt $\eta_{\max }$ mit zunehmender Partikelgröße an. Zudem ist ein Einfluss der Partikelkonzentration festzustellen. Für die Rückstreuamplitude kann ein Vergleich mit etablierten Messverfahren (ähnlich der Vorgehensweise bei der Dämpfung) nicht durchgeführt werden, da ein entsprechendes Referenzmessverfahren/-gerät nicht verfügbar ist. Alternativ dazu erlaubt ein Vergleich mit Modellvorhersagen eine Plausibilitätsprüfung. Das für die Schallstreuung an Partikeln entwickelte Modell nach Faran bestätigt qualitativ den Anstieg der (Rück)Streuamplitude mit zunehmender Partikelgröße so wie es qualitativ auch in Abb. 6 durch Messungen gezeigt werden kann $[6,7]$. 


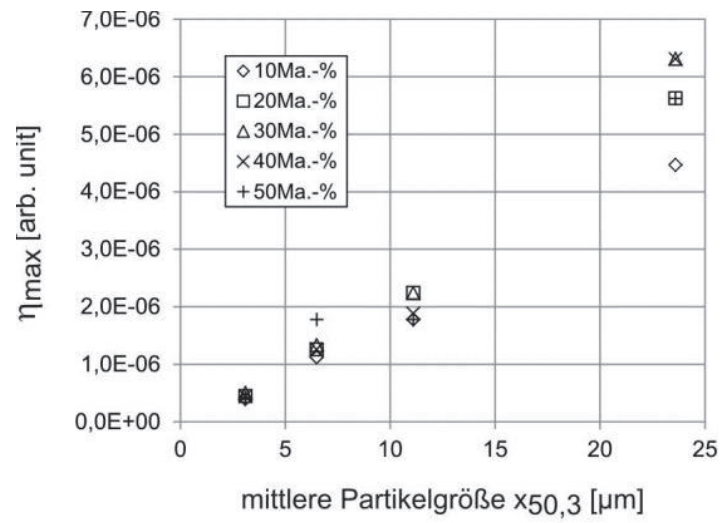

Abb. 6. Maximalwert der Rückstreuamplitude $\eta_{\max }$ als Funktion der mittleren Partikelgröße bei verschiedenen Partikelkonzentrationen $(f=10 \mathrm{MHz})$.

\section{Zusammenfassung}

Das vorgestellte Verfahren auf Basis der Ultraschallrückstreumessung an bewegten Partikelsystemen bietet eine Erweiterung zur bislang angewendeten Ultraschall-Dämpfungsspektroskopie im Bereich der akustischen Partikelcharakterisierung. Die statistische Auswertung der laufzeitabhängigen Rückstreusignale liefert zwei unabhängige Parameter $\eta_{\max }$ und $\Delta \eta$, die (im Rahmen der hier untersuchten Schallfrequenzen, Partikelgrößen und -konzentrationen) mit den Eigenschaften der Partikelkonzentration und -größe korrelieren.

Die Vermeidung eines Messspalts, wie im Fall der klassischen Transmissionsanordnung, erhöht das Potenzial des Messverfahrens zur Inline-Anwendung und ist vor allem für den Einsatz bei hohen Partikelkonzentrationen prädestiniert. Die offene Messanordnung erlaubt die Konstruktion einer einfachen Stabsonde, die in die Suspension eingetaucht oder direkt in $\mathrm{Be}-$ hälterwandungen eingebaut wird.

Die Ergebnisse zeigen, dass Partikel im unteren Mikrometermaßstab auch bei schwacher Schallstreuung auswertbare Signale liefern. Grundsätzlich wird die detektierbare Partikelgröße von der verwendeten Schallfrequenz bestimmt, wobei kleinere Partikel eine höhere Frequenz (kleinere Wellenlänge) erfordern. Mit Schallfrequenzen größer $20 \mathrm{MHz}$ lassen sich submikrone Partikel prinzipiell vermessen. Größere Partikel liefern vergleichsweise stärkere Rückstreusignale, so dass der mit dem Verfahren detektierbare Partikelgrößenbereich nach oben keine Beschränkung aufweist.

Mögliche Zielanwendungen der Messmethode sind Prozesse mit flüssigkeitsgetragenen Partikelsystemen, deren Konzentration von wenigen bis hin zu 50 Ma.-\% (hochgefüllte Suspensionen) reicht. Hierzu zählen beispielsweise Nasszerkleinerungsprozesse, wie sie bei der
Herstellung von Farben und Lacken oder bei der Aufbereitung von Erz- und Klärschlämmen zu finden sind. Im Sinne eines ProzessMonitoring ist eine Korrelation zwischen akustischer Messgröße und Zielgröße (hier: Partikelgröße) oftmals schon ausreichend, um eine entsprechende Prozesssteuerung, wie zum Beispiel die Mahlpunktenderkennung umzusetzen. Ferner ist in der Halbleiterfertigung das Monitoring von hochgefüllten Säge- und Schleifsuspensionen zur Qualitätssicherung des Herstellungsprozesses ebenso ein potenzielles Anwendungsfeld für diese Form der akustischen Partikelmesstechnik.

\section{Förderung}

Das IGF-Vorhaben 16681 BR der Forschungsvereinigung Forschungsgesellschaft für Messtechnik, Sensorik und Medizintechnik e.V. Dresden - fms, Theodor-Heuss-Allee 25, 60486 Frankfurt am Main wurde über die AiF im Rahmen des Programms zur Förderung der industriellen Gemeinschaftsforschung und entwicklung (IGF) vom Bundesministerium für Wirtschaft und Technologie aufgrund eines Beschlusses des Deutschen Bundestages gefördert.

\section{Literatur}

[1] H. Alrutz, Über die Anwendung von Pseudorauschfolgen zur Messung an linearen Übertragungssystemen, Dissertation, Georg-August Universität Göttingen, 1983

[2] R. Millner, Wissensspeicher Ultraschalltechnik, 1. Aufl. Leipzig: VEB Fachbuchverlag 1987

[3] A.S. Dukhin, P.J. Goetz, Ultrasound for Characterizing Colloids, Elsevier, 2002

[4] R. Weser, B. Wessely, S. Wöckel, U. Hempel, Partikelcharakterisierung in hochkonzentrierten Dispersionen - Grundlegende Untersuchungen zu einer neuen Ultraschallmethode, Chemie Ingenieur Technik, Volume 85, Issue 5 (2013), pages 753-757

[5] DT100 / DT1200 Ultraschallspektrometer, Dispersion Technology, Inc., http://dispersion.com

[6] J.J. Faran, Sound scattering by solid cylinders and spheres, J. Acoust. Soc. Am. 1951, 23, 405418

[7] R. Weser, S. Wöckel, B. Wessely, U. Hempel, Particle characterisation in highly concentrated dispersions using ultrasonic backscattering method, Ultrasonics 53 (2012) 706-716 